\title{
Iterative algorithms for finding a common solution of system of the set of variational inclusion problems and the set of fixed point problems
}

\author{
Atid Kangtunyakarn
}

Correspondence: beawrock@hotmail.com Department of Mathematics, Faculty of Science, King Mongkut's Institute of Technology Ladkrabang, Bangkok 10520, Thailand

\begin{abstract}
In this article, we introduce a new mapping generated by infinite family of nonexpansive mapping and infinite real numbers. By means of the new mapping, we prove a strong convergence theorem for finding a common element of the set of fixed point problems of infinite family of nonexpansive mappings and the set of a finite family of variational inclusion problems in Hilbert space. In the last section, we apply our main result to prove a strong convergence theorem for finding a common element of the set of fixed point problems of infinite family of strictly pseudocontractive mappings and the set of finite family of variational inclusion problems.
\end{abstract}

Keywords: nonexpansive mapping, strict pseudo contraction, strongly positive operator, variational inclusion problem, fixed point

\section{Introduction}

Let $H$ be a real Hilbert space and let $C$ be a nonempty closed convex subset of $\mathrm{H}$. Let $A$ : $C \rightarrow H$ be a nonlinear mapping and let $F: C \times C \rightarrow \mathbb{R}$ be a bifunction. A mapping $T$ of $H$ into itself is called nonexpansive if $\|T x-T y\| \leq\|x-y\|$ for all $x, y \in H$. We denote by $F$ (T) the set of fixed points of $T$ (i.e. $F(T)=\{x \in H: T x=x\}$ ). Goebel and Kirk [1] showed that $F(T)$ is always closed convex and also nonempty provided $T$ has a bounded trajectory.

The problem for finding a common fixed point of a family of nonexpansive mappings has been studied by many authors. The well-known convex feasibility problem reduces to finding a point in the intersection of the fixed point sets of a family of nonexpansive mappings (see, e.g., [2,3]).

A bounded linear operator $A$ on $H$ is called strongly positive with coefficient $\bar{\gamma}$ if there exists a constant $\bar{\gamma}>0$ with the property

$$
\langle A x, x\rangle \geq \bar{\gamma}\|x\|^{2} .
$$

A mapping $A$ of $C$ into $H$ is called inverse-strongly monotone, see [4], if there exists a positive real number $\alpha$ such that

$$
\langle x-y, A x-A y\rangle \geq \alpha\|A x-A y\|^{2}
$$

for all $x, y \in C$.

The variational inequality problem is to find a point $u \in C$ such that

$$
\langle v-u, A u\rangle \geq 0 \quad \text { for all } v \in C .
$$

(C) 2011 Kangtunyakarn; licensee Springer. This is an Open Access article distributed under the terms of the Creative Commons Attribution License (http://creativecommons.org/licenses/by/2.0), which permits unrestricted use, distribution, and reproduction in any medium, provided the original work is properly cited. 
The set of solutions of (1.1) is denoted by $V I(C, A)$. Many authors have studied methods for finding solution of variational inequality problems (see, e.g., [5-8]).

In 2008, Qin et al. [9] introduced the following iterative scheme:

$$
\left\{\begin{array}{l}
y_{n}=P_{C}\left(I-s_{n} A\right) x_{n} \\
x_{n+1}=\alpha_{n} \gamma f\left(W_{n} x_{n}\right)+\left(I-\alpha_{n} B\right) W_{n} P_{C}\left(I-r_{n} A\right) y_{n}, \quad \forall n \in \mathbb{N},
\end{array}\right.
$$

where $W_{n}$ is the $W$-mapping generated by a finite family of nonexpansive mappings and real numbers, $A: C \rightarrow H$ is relaxed (u,v) cocoercive and $\mu$-Lipschitz continuous, and $P_{C}$ is a metric projection $H$ onto $C$. Under suitable conditions of $\left\{s_{n}\right\},\left\{r_{n}\right\}\left\{\alpha_{n}\right\}, \gamma$, they proved that $\left\{x_{n}\right\}$ converges strongly to an element of the set of variational inequality problem and the set of a common fixed point of a finite family of nonexpansive mappings.

In 2006, Marino and $\mathrm{Xu}$ [10] introduced the iterative scheme as follows:

$$
x_{0} \in H, x_{n+1}=\left(I-\alpha_{n} A\right) S x_{n}+\alpha_{n} \gamma f\left(x_{n}\right), \quad \forall n \geq 0,
$$

where $S$ is a nonexpansive mapping, $f$ is a contraction with the coefficient $a \in(0,1)$, $A$ is a strongly positive bounded linear self-adjoint operator with the coefficient $\bar{\gamma}$, and $\gamma$ is a constant such that $0<\gamma<\frac{\bar{\gamma}}{a}$. They proved that $\left\{x_{n}\right\}$ generated by the above iterative scheme converges strongly to the unique solution of the variational inequality:

$$
\left\langle(A-\gamma f) x^{*}, x-x^{*}\right\rangle \geq 0, x \in F(S) .
$$

We know that a mapping $B: H \rightarrow H$ is said to be monotone, if for each $x, y \in H$, we have

$$
\langle B x-B y, x-y\rangle \geq 0 .
$$

A set-valued mapping $M: H \rightarrow 2^{H}$ is called monotone if for all $x, y \in H, f \in M x$ and $g \in M y$ imply $\langle x-y, f-g\rangle \geq 0$. A monotone mapping $M: H \rightarrow 2^{H}$ is maximal if the graph of $\operatorname{Graph}(M)$ of $\mathrm{M}$ is not properly contained in the graph of any other monotone mapping. It is known that a monotone mapping $M$ is maximal if and only if for $(x, f) \in$ $H \times H,\langle x-y, f-g\rangle \geq 0$ for every $(y, g) \in \operatorname{Graph}(M)$ implies $f \in M x$.

Next, we consider the following so-called variational inclusion problem:

Find a $u \in H$ such that

$$
\theta \in B u+M u
$$

where $B: H \rightarrow H, M: H \rightarrow 2^{H}$ are two nonlinear mappings, and $\theta$ is zero vector in $H$ (see, for instance, [11-16]). The set of the solution of (1.4) is denoted by $V I(H, B, M)$.

Let $C$ be a nonempty closed convex subset of Banach space $X$. Let $\left\{T_{n}\right\}_{n=1}^{\infty}$ be an infinite family of nonexpansive mappings of $C$ into itself, and let $\lambda_{1}, \lambda_{2}, \ldots$, be real numbers in $[0,1]$; then we define the mapping $K_{n}: C \rightarrow C$ as follows:

$$
\begin{aligned}
U_{n, 0} & =I \\
U_{n, 1} & =\lambda_{1} T_{1} U_{n, 0}+\left(1-\lambda_{1}\right) U_{n, 0}, \\
U_{n, 2} & =\lambda_{2} T_{2} U_{n, 1}+\left(1-\lambda_{2}\right) U_{n, 1} \\
U_{n, 3} & =\lambda_{3} T_{3} U_{n, 2}+\left(1-\lambda_{3}\right) U_{n, 2} \\
& \vdots \\
U_{n, k} & =\lambda_{k} T_{k} U_{n, k-1}+\left(1-\lambda_{k}\right) U_{n, k-1} \\
U_{n, k+1} & =\lambda_{k+1} T_{k+1} U_{n, k}+\left(1-\lambda_{k+1}\right) U_{n, k} \\
& \vdots \\
U_{n, n-1} & =\lambda_{n-1} T_{n-1} U_{n, n-2}+\left(1-\lambda_{n-1}\right) U_{n, n-2} \\
K_{n}= & U_{n, n}=\lambda_{n} T_{n} U_{n, n-1}+\left(1-\lambda_{n}\right) U_{n, n-1} .
\end{aligned}
$$


Such a mapping $K_{n}$ is called the $K$-mapping generated by $T_{1}, T_{2}, \ldots, T_{n}$ and $\lambda_{1}, \lambda_{2}, \ldots, \lambda_{n}$. Let $x_{1} \in H$ and $\left\{x_{n}\right\}$ be the sequence generated by

$$
x_{n+1}=\alpha_{n} \gamma f\left(x_{n}\right)+\beta_{n} x_{n}+\left(\left(1-\beta_{n}\right) I-\alpha_{n} A\right)\left(\gamma_{n} K_{n} x_{n}+\left(1-\gamma_{n}\right) S x_{n}\right),
$$

where $A$ is a strongly positive linear-bounded self-adjoint operator with the coefficient $0<\bar{\gamma}<1, S: C \rightarrow C$ is the $S$ - mapping generated by $G_{1}, G_{2}, \ldots, G_{N}$ and $v_{1}, v_{2}, \ldots$, $v_{N}$, where $G_{i}: H \rightarrow H$ is a mapping defined by $J_{M_{i, n}}\left(I-\eta B_{i}\right) x=G_{i} x$ for every $x \in H$, and $\eta \in\left(0,2 \delta_{i}\right)$ for every $i=1,2, \ldots, N, f: H \rightarrow H$ is contractive mapping with coeffi-

cient $\theta \in(0,1)$ and $0<\gamma<\frac{\bar{\gamma}}{\theta},\left\{\alpha_{n}\right\},\left\{\beta_{n}\right\},\left\{\gamma_{n}\right\}$ are sequences in $[0,1]$.

In this article, by motivation of (1.3), we prove a strong convergence theorem of the proposed algorithm scheme (1.5) to an element $z \in \bigcap_{i=1}^{\infty} F\left(T_{i}\right) \bigcap \bigcap_{i=1}^{N} V\left(H, B_{i}, M_{i}\right)$, under suitable conditions of $\left\{\alpha_{n}\right\},\left\{\beta_{n}\right\},\left\{\gamma_{n}\right\}$.

\section{Preliminaries}

In this section, we provide some useful lemmas that will be used for our main result in the next section.

Let $C$ be a closed convex subset of a real Hilbert space $H$, and let $P_{C}$ be the metric projection of $H$ onto $C$, i.e., for $x \in H, P_{C} x$ satisfies the property:

$$
\left\|x-P_{C} x\right\|=\min _{y \in C}\|x-y\| .
$$

The following characterizes the projection $P_{C}$.

Lemma 2.1. (see [17]) Given $x \in H$ and $y \in C$. Then $P_{C} x=y$ if and only if there holds the inequality

$$
\langle x-y, y-z\rangle \geq 0 \quad \forall z \in C .
$$

Lemma 2.2. (see [18]) Let $\left\{s_{n}\right\}$ be a sequence of nonnegative real number satisfying

$$
s_{n+1}=\left(1-\alpha_{n}\right) s_{n}+\alpha_{n} \beta_{n}, \quad \forall n \geq 0
$$

where $\left\{\alpha_{n}\right\},\left\{\beta_{n}\right\}$ satisfy the conditions:

(1) $\left\{\alpha_{n}\right\} \subset[0,1], \sum_{n=1}^{\infty} \alpha_{n}=\infty$;

(2) $\limsup _{n \rightarrow \infty} \beta_{n} \leq 0$ or $\sum_{n=1}^{\infty}\left|\alpha_{n} \beta_{n}\right|<\infty$.

Then $\lim _{n \rightarrow \infty} s_{n}=0$.

Lemma 2.3. (see [19]) Let $C$ be a closed convex subset of a strictly convex Banach space E. Let $\left\{T_{n}: n \in \mathbb{N}\right\}$ be a sequence of nonexpansive mappings on $C$. Suppose $\bigcap_{n=1}^{\infty} F\left(T_{n}\right)$ is nonempty. Let $\left\{\lambda_{n}\right\}$ be a sequence of positive numbers with $\Sigma_{n=1}^{\infty} \lambda_{n}=1$. Then a mapping $S$ on $C$ defined by

$$
S(x)=\sum_{n=1}^{\infty} \lambda_{n} T_{n} x_{n}
$$

for $x \in C$ is well defined, nonexpansive and $F(S)=\bigcap_{n=1}^{\infty} F\left(T_{n}\right)$ hold.

Lemma 2.4. (see [20]) Let $E$ be a uniformly convex Banach space, $C$ be a nonempty closed convex subset of $E$, and $S: C \rightarrow C$ be a nonexpansive mapping. Then $I-S$ is demi-closed at zero. 
Lemma 2.5. (see [21]) Let $\left\{x_{n}\right\}$ and $\left\{z_{n}\right\}$ be bounded sequences in a Banach space $X$ and let $\left\{\beta_{n}\right\}$ be a sequence in $[0,1]$ with $0<\lim \inf _{n \rightarrow \infty} \beta_{n} \leq \lim \sup _{n \rightarrow \infty} \beta_{n}<1$.

Suppose $x_{n+1}=\beta_{n} x_{n}+\left(1-\beta_{n}\right) z_{n}$ for all integer $n \geq 0$ and $\lim _{\sup _{n \rightarrow \infty}}\left(\mid z_{n+1}-z_{n}\|-\|\right.$ $\left.x_{n+1}-x_{n}||\right) \leq 0$. Then $\lim _{n \rightarrow \infty}|| x_{n}-z_{n} \|=0$.

In 2009, Kangtunykarn and Suantai [5] introduced the $S$-mapping generated by a finite family of nonexpansive mappings and real numbers as follows:

Definition 2.1. Let $C$ be a nonempty convex subset of real Banach space. Let $\left\{T_{i}\right\}_{i=1}^{N}$ be a finite family of nonexpanxive mappings of $C$ into itself. For each $j=1,2, \ldots, N$, let $\alpha_{j}=\left(\alpha_{1}^{j}, \alpha_{2}^{j}, \alpha_{3}^{j}\right) \in I \times I \times$ Iwhere $I \in[0,1]$ and $\alpha_{1}^{j}+\alpha_{2}^{j}+\alpha_{3}^{j}=1$, define the mapping $S:$ $C \rightarrow C$ as follows:

$$
\begin{aligned}
U_{0}= & I \\
U_{1}= & \alpha_{1}^{1} T_{1} U_{0}+\alpha_{2}^{1} U_{0}+\alpha_{3}^{1} I \\
U_{2}= & \alpha_{1}^{2} T_{2} U_{1}+\alpha_{2}^{2} U_{1}+\alpha_{3}^{2} I \\
U_{3}= & \alpha_{1}^{3} T_{3} U_{2}+\alpha_{2}^{3} U_{2}+\alpha_{3}^{3} I \\
& \vdots \\
U_{N-1}= & \alpha_{1}^{N-1} T_{N-1} U_{N-2}+\alpha_{2}^{N-1} U_{N-2}+\alpha_{3}^{N-1} I \\
S & =U_{N}=\alpha_{1}^{N} T_{N} U_{N-1}+\alpha_{2}^{N} U_{N-1}+\alpha_{3}^{N} I .
\end{aligned}
$$

This mapping is called the S-mapping generated by $T_{1}, \ldots, T_{N}$ and $\alpha_{1}, \alpha_{2}, \ldots, \alpha_{N}$.

Lemma 2.6. (see [5]) Let $C$ be a nonempty closed convex subset of strictly convex. Let $\left\{T_{i}\right\}_{i=1}^{N}$ be a finite family of nonexpanxive mappings of $C$ into itself with $\bigcap_{i=1}^{N} F\left(T_{i}\right) \neq$ Øand let $\alpha_{j}=\left(\alpha_{1}^{j}, \alpha_{2}^{j}, \alpha_{3}^{j}\right) \in I \times I \times I, j=1,2,3, \ldots, N$, where $I=[0,1]$, $\alpha_{1}^{j} \in(0,1), \alpha_{1}^{j} \in(0,1)$ for all $\mathrm{j}=1,2, \ldots, \mathrm{N}-1, \alpha_{1}^{N} \in(0,1] \alpha_{2}^{j}, \alpha_{3}^{j} \in[0,1)$ for all $\mathrm{j}=1,2, \ldots$, $\mathrm{N}$. Let $S$ be the mapping generated by $T_{1}, \ldots, T_{N}$ and $\alpha_{1}, \alpha_{2}, \ldots, \alpha_{N}$. Then $F(S)=\bigcap_{i=1}^{N} F\left(T_{i}\right)$.

Lemma 2.7. (see [5]) Let $C$ be a nonempty closed convex subset of Banach space. Let $\left\{T_{i}\right\}_{i=1}^{N}$ be a finite family of nonexpansive mappings of $C$ into itself and $\alpha_{j}=\left(\alpha_{1}^{j}, \alpha_{2}^{j}, \alpha_{3}^{j}\right) \in I \times I \times I, \quad \alpha_{j}=\left(\alpha_{1}^{j}, \alpha_{2}^{j}, \alpha_{3}^{j}\right) \in I \times I \times I, \quad$ where $I=[0,1]$, $\alpha_{1}^{j}+\alpha_{2}^{j}+\alpha_{3}^{j}=1$ and $\alpha_{1}^{j}+\alpha_{2}^{j}+\alpha_{3}^{j}=1$ such that $\alpha_{i}^{n, j} \rightarrow \alpha_{i}^{j} \in[0,1]$ as $n \rightarrow \infty$ for $i=1,3$ and $j=1,2,3, \ldots, N$. Moreover, for every $n \in \mathbb{N}$, let $S$ and $S_{n}$ be the $S$-mappings generated by $T_{1}, T_{2}, \ldots, T_{N}$ and $\alpha_{1}, \alpha_{2}, \ldots, \alpha_{N}$ and $T_{1}, T_{2}, \ldots, T_{N}$ and $\alpha_{1}^{(n)}, \alpha_{2}^{(n)}, \ldots, \alpha_{N}^{(n)}$, respectively. Then $\lim _{n \rightarrow \infty}\left\|S_{n} x-S x\right\|=0$ for every $x \in C$.

Definition 2.2. (see [11]) Let $M: H \rightarrow 2^{H}$ be a multi-valued maximal monotone mapping, then the single-valued mapping $J_{M, \lambda}: H \rightarrow H$ defined by

$$
J_{M, \lambda}(u)=(I+\lambda M)^{-1}(u), \quad \forall u \in H,
$$

is called the resolvent operator associated with $M$, where $\lambda$ is any positive number and I is identity mapping.

Lemma 2.8. (see [11]) $u \in H$ is a solution of variational inclusion (1.4) if and only if $u=J_{M, \lambda}(u-\lambda B u), \forall \lambda>0$, i.e.,

$$
V I(H, B, M)=F\left(J_{M, \lambda}(I-\lambda B)\right), \quad \forall \lambda>0 .
$$


Further, if $\lambda \in(0,2 \alpha]$, then $V I(H, B, M)$ is closed convex subset in $H$.

Lemma 2.9. (see [22]) The resolvent operator $J_{M, \lambda}$ associated with $M$ is single-valued, nonexpansive for all $\lambda>0$ and 1-inverse-strongly monotone.

Lemma 2.10. In a strictly convex Banach space E, if

$$
\|x\|=\|y\|=\|\lambda x+(1-\lambda) y\|
$$

for all $x, y \in E$ and $\lambda \in(0,1)$, then $x=y$.

Lemma 2.11. Let $C$ be a nonempty closed convex subset of a strictly convex Banach space. Let $\left\{T_{i}\right\}_{i=1}^{\infty}$ be an infinite family of nonexpanxive mappings of $C$ into itself with $\bigcap_{i=1}^{\infty} F\left(T_{i}\right) \neq \emptyset$ and let $\lambda_{1}, \lambda_{2}, \ldots$, be real numbers such that $0<\lambda_{i}<1$ for every $i=1,2, \ldots$, and $\sum_{i=1}^{\infty} \lambda_{i}<\infty$. For every $n \in \mathbb{N}$, let $K_{n}$ be the K-mapping generated by $T_{1}, T_{2}, \ldots, T_{n}$ and $\lambda_{1}, \lambda_{2}, \ldots, \lambda_{n}$. Then for every $x \in C$ and $k \in \mathbb{N}, \lim _{n \rightarrow \infty} K_{n} x$ exits.

Proof. Let $x \in C$. Then for $k, n \in \mathbb{N}$, we have

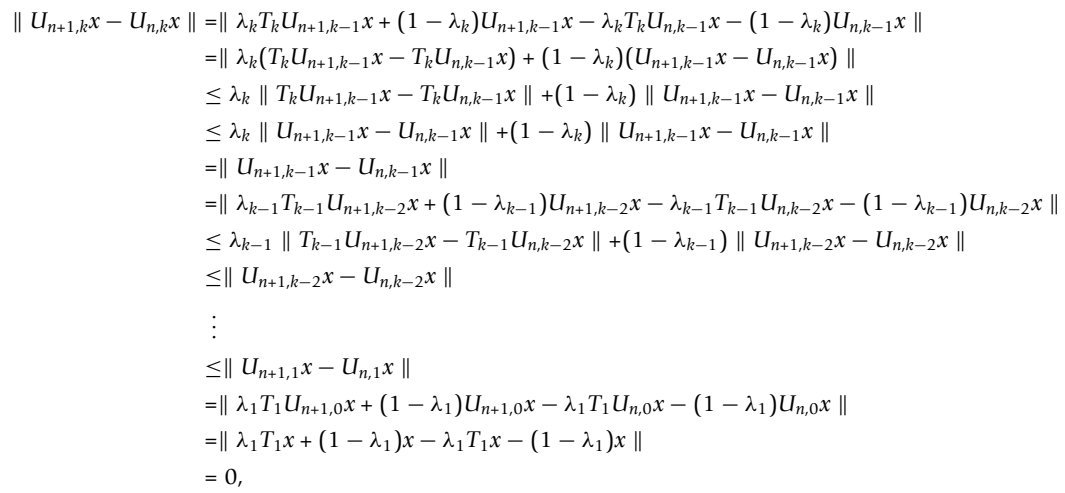

which implies that $U_{n+1, k}=U_{n, k}$ for every $k, n \in \mathbb{N}$. Hence, $K_{n}=U_{n, n}=U_{n+1, n}$. Since $K_{n+1} x=U_{n+1, n+1} x=\lambda_{n+1} T_{n+1} K_{n} x+\left(1-\lambda_{n+1}\right) K_{n} x$, we have

$$
K_{n+1} x-K_{n} x=\lambda_{n+1}\left(T_{n+1} K_{n} x-K_{n} x\right) .
$$

Let $x^{*} \in \bigcap_{i=1}^{\infty} F\left(T_{i}\right)$ and $x \in C$. For each $n \in \mathbb{N}$, we have

$$
\begin{aligned}
\left\|K_{n} x-x^{*}\right\| & =\left\|\lambda_{n} T_{n} U_{n, n-1} x+\left(1-\lambda_{n}\right) U_{n, n-1} x-x^{*}\right\| \\
& \leq \lambda_{n}\left\|T_{n} U_{n, n-1} x-x^{*}\right\|+\left(1-\lambda_{n}\right)\left\|U_{n, n-1} x-x^{*}\right\| \\
& \leq\left\|U_{n, n-1} x-x^{*}\right\| \\
& =\left\|\lambda_{n-1} T_{n-1} U_{n, n-2} x+\left(1-\lambda_{n-1}\right) U_{n, n-2} x-x^{*}\right\| \\
& \leq \lambda_{n-1}\left\|T_{n-1} U_{n, n-2} x-x^{*}\right\|+\left(1-\lambda_{n-1}\right)\left\|U_{n, n-2} x-x^{*}\right\| \\
& \leq\left\|U_{n, n-2} x-x^{*}\right\| \\
& \cdot \\
& \cdot \\
& \cdot \\
& \leq\left\|U_{n, 1} x-x^{*}\right\| \\
& =\left\|\lambda_{1} T_{1} U_{n, 0} x+\left(1-\lambda_{1}\right) U_{n, 0} x-x^{*}\right\| \\
& \leq \lambda_{1}\left\|T_{1} x-x^{*}\right\|+\left(1-\lambda_{1}\right)\left\|x-x^{*}\right\| \\
& =\left\|x-x^{*}\right\|,
\end{aligned}
$$


which implies that $\left\{K_{n} x\right\}$ is bounded, and so is $\left\{T_{n} K_{n} x\right\}$. For $m \geq n$, by (2.3) we have

$$
\begin{aligned}
\left\|K_{m} x-K_{n} x\right\|= & \| K_{m} x-K_{m-1} x+K_{m-1} x-K_{m-2} x+K_{m-2} x-+\cdots \\
& -K_{n+1} x+K_{n+1} x-K_{n} x \| \\
\leq & \left\|K_{m} x-K_{m-1} x\right\|+\left\|K_{m-1} x-K_{m-2} x\right\|+\left\|K_{m-2} x-K_{m-3} x\right\|+\cdots \\
& +\left\|K_{n+2}-K_{n+1} x\right\|+\left\|K_{n+1} x-K_{n} x\right\| \\
= & \lambda_{m}\left\|T_{m} K_{m-1} x-K_{m-1} x\right\|+\lambda_{m-1}\left\|T_{m-1} K_{m-2} x-K_{m-2} x\right\|+\cdots \\
& +\lambda_{n+1}\left\|T_{n+1} K_{n} x-K_{n} x\right\| \\
\leq & M \sum_{k=n+1}^{m} \lambda_{k}
\end{aligned}
$$

where $M=\sup _{n \in \mathbb{N}}\left\{|| \mathrm{T}_{n+1} K_{n} x-K_{n} x \|\right\}$. This implies that $\left\{K_{n} x\right\}$ is Cauchy sequence. Hence $\lim _{n \rightarrow \infty} K_{n} x$ exists.

From Lemma 2.11, we can define a mapping $K: C \rightarrow C$ as follows:

$$
K x=\lim _{n \rightarrow \infty} K_{n} x, \quad x \in C .
$$

Such a mapping $K$ is called the $K$-mapping generated by $T_{1}, T_{2}, \ldots$, and $\lambda_{1}, \lambda_{2}, \ldots$...

Remark 2.12. It is easy to see that for each $n \in \mathbb{N}, K_{n}$ is nonexpansive mappings. Let $x, y \in C$, then we have

$$
\|K x-K y\|=\lim _{n \Rightarrow \infty}\left\|K_{n} x-K_{n} y\right\| \leq\|x-y\| .
$$

By (2.6), we have $K: C \rightarrow C$ is nonexpansive mapping. Next, we will show that $\lim _{n \rightarrow \infty} \sup _{x \in D}\left\|K_{n} x-K x\right\|=0$ for every bounded subset $D$ of $C$. To show this, let $x, y$ $\in C$ and $D$ be a bounded subset of $C$. By (2.5), for $m \geq n$, we have

$$
\left\|K_{m} x-K_{n} x\right\| \leq M \sum_{k=n+1}^{m} \lambda_{k} .
$$

By letting $m \rightarrow \infty$, for any $x \in D$, we have

$$
\left\|K x-K_{n} x\right\| \leq M \sum_{k=n+1}^{\infty} \lambda_{k} .
$$

Since $\sum_{n=1}^{\infty} \lambda_{n}<\infty$, we have

$$
\lim _{n \rightarrow \infty} \sup _{x \in D}\left\|K x-K_{n} x\right\|=0 .
$$

By the next lemma, we will show that $F(K)=\bigcap_{i=1}^{\infty} F\left(T_{i}\right)$

Lemma 2.13. Let $C$ be a nonempty closed convex subset of a strictly convex Banach space. Let $\left\{T_{i}\right\}_{i=1}^{\infty}$ be an infinite family of nonexpansive mappings of $C$ into itself with $\bigcap_{i=1}^{\infty} F\left(T_{i}\right) \neq \emptyset$, and let $\lambda_{1}, \lambda_{2}, \ldots$, be real numbers such that $0<\lambda_{i}<1$ for every $i=1,2, \ldots$ with $\sum_{i=1}^{\infty} \lambda_{i}<\infty$. Let $K_{n}$ and $K$ be the K-mapping generated by $T_{1}, T_{2}, \ldots T_{n}$ and $\lambda_{1}$, $\lambda_{2}, \ldots \lambda_{n}$ and $T_{1}, T_{2}, \ldots$ and $\lambda_{1}, \lambda_{2}, \ldots$, respectively. Then $F(K)=\bigcap_{i=1}^{\infty} F\left(T_{i}\right)$.

Proof. It is easy to see that $\bigcap_{i=1}^{\infty} F\left(T_{i}\right) \subseteq F(K)$. Next, we show that $F(K) \subseteq \bigcap_{i=1}^{\infty} F\left(T_{i}\right)$. Let $x_{0} \in F(K)$ and $x^{*} \in \bigcap_{i=1}^{\infty} F\left(T_{i}\right)$. Let $k \in \mathbb{N}$ be fixed. Since 


$$
\begin{aligned}
\left\|K_{n} x_{0}-x^{*}\right\| & =\left\|\lambda_{n} T_{n} U_{n, n-1} x_{0}+\left(1-\lambda_{n}\right) U_{n, n-1} x_{0}-x^{*}\right\| \\
& =\left\|\lambda_{n}\left(T_{n} U_{n, n-1} x_{0}-x^{*}\right)+\left(1-\lambda_{n}\right)\left(U_{n, n-1} x_{0}-x^{*}\right)\right\| \\
& \leq \lambda_{n}\left\|T_{n} U_{n, n-1} x_{0}-x^{*}\right\|+\left(1-\lambda_{n}\right)\left\|U_{n, n-1} x_{0}-x^{*}\right\| \\
& \leq\left\|U_{n, n-1} x_{0}-x^{*}\right\| \\
& =\left\|\lambda_{n-1}\left(T_{n-1} U_{n, n-2} x_{0}-x^{*}\right)+\left(1-\lambda_{n-1}\right) U_{n, n-2}\left(x_{0}-x^{*}\right)\right\| \\
& \leq \lambda_{n-1}\left\|T_{n-1} U_{n, n-2} x_{0}-x^{*}\right\|+\left(1-\lambda_{n-1}\right)\left\|U_{n, n-2} x_{0}-x^{*}\right\| \\
& \leq\left\|U_{n, n-2} x_{0}-x^{*}\right\| \\
& \vdots \\
& \cdot \\
& \cdot \\
& \leq\left\|U_{n, k} x_{0}-x^{*}\right\| \\
& =\left\|\lambda_{k}\left(T_{k} U_{n, k-1} x_{0}-x^{*}\right)+\left(1-\lambda_{k}\right)\left(U_{n, k-1} x_{0}-x^{*}\right)\right\| \\
& \leq \lambda_{k}\left\|T_{k} U_{n, k-1} x_{0}-x^{*}\right\|+\left(1-\lambda_{k}\right)\left\|U_{n, k-1} x_{0}-x^{*}\right\| \\
& \leq\left\|U_{n, k-1} x_{0}-x^{*}\right\| \\
& \cdot \\
& \cdot \\
& \cdot \\
& \leq\left\|U_{n, 1} x_{0}-x^{*}\right\| \\
& =\left\|\lambda_{1}\left(T_{1} x_{0}-x^{*}\right)+\left(1-\lambda_{1}\right)\left(x_{0}-x^{*}\right)\right\| \\
& \leq \lambda_{1}\left\|T_{1} x_{0}-x^{*}\right\|+\left(1-\lambda_{1}\right)\left\|x_{0}-x^{*}\right\| \\
& \leq\left\|x_{0}-x^{*}\right\|,
\end{aligned}
$$

we have

$$
\begin{aligned}
\left\|x_{0}-x^{*}\right\| & =\lim _{n \rightarrow \infty}\left\|K_{n} x_{0}-x^{*}\right\| \leq\left\|\lambda_{1}\left(T_{1} x_{0}-x^{*}\right)+\left(1-\lambda_{1}\right)\left(x_{0}-x^{*}\right)\right\| \\
& \leq \lambda_{1}\left\|T_{1} x_{0}-x^{*}\right\|+\left(1-\lambda_{1}\right)\left\|x_{0}-x^{*}\right\| \\
& \leq\left\|x_{0}-x^{*}\right\|
\end{aligned}
$$

this implies that

$$
\left\|x_{0}-x^{*}\right\|=\left\|T_{1} x_{0}-x^{*}\right\|=\left\|\lambda_{1}\left(T_{1} x_{0}-x^{*}\right)+\left(1-\lambda_{1}\right)\left(x_{0}-x^{*}\right)\right\| .
$$

By Lemma 2.10, we have $T_{1} x_{0}=x_{0}$, that is $x_{0} \in F\left(T_{1}\right)$. It follows that $U_{n, 1} x_{0}=x_{0}$. By (2.7), we have

$$
\begin{aligned}
\left\|K_{n} x_{0}-x^{*}\right\| & \leq\left\|U_{n, 2} x_{0}-x^{*}\right\|=\left\|\lambda_{2}\left(T_{2} U_{n, 1} x_{0}-x^{*}\right)+\left(1-\lambda_{2}\right)\left(U_{n, 1} x_{0}-x^{*}\right)\right\| \\
& =\left\|\lambda_{2}\left(T_{2} x_{0}-x^{*}\right)+\left(1-\lambda_{2}\right)\left(x_{0}-x^{*}\right)\right\| \\
& \leq \lambda_{2}\left\|T_{2} x_{0}-x^{*}\right\|+\left(1-\lambda_{2}\right)\left\|x_{0}-x^{*}\right\| \\
& \leq\left\|x_{0}-x^{*}\right\| .
\end{aligned}
$$

It follows that

$$
\begin{aligned}
\left\|x_{0}-x^{*}\right\| & =\lim _{n \rightarrow \infty}\left\|K_{n} x_{0}-x^{*}\right\| \\
& \leq\left\|\lambda_{2}\left(T_{2} x_{0}-x^{*}\right)+\left(1-\lambda_{2}\right)\left(x_{0}-x^{*}\right)\right\| \\
& \leq \lambda_{2}\left\|T_{2} x_{0}-x^{*}\right\|+\left(1-\lambda_{2}\right)\left\|x_{0}-x^{*}\right\| \\
& \leq\left\|x_{0}-x^{*}\right\|,
\end{aligned}
$$


which implies

$$
\left\|x_{0}-x^{*}\right\|=\left\|T_{2} x_{0}-x^{*}\right\|=\left\|\lambda_{2}\left(T_{2} x_{0}-x^{*}\right)+\left(1-\lambda_{2}\right)\left(x_{0}-x^{*}\right)\right\| .
$$

By Lemma 2.10, we obtain that $T_{2} x_{0}=x_{0}$, that is $x_{0} \in F\left(T_{2}\right)$. It follows that $U_{n, 2} x_{0}=$ $x_{0}$. By using the same argument, we can conclude that $T_{i} x_{0}=x_{0}$ and $U_{i} x_{0}=x_{0}$ for $i=$ $1,2, \ldots, k-1$. By (2.7), we have

$$
\begin{aligned}
\left\|K_{n} x_{0}-x^{*}\right\| & \leq\left\|U_{n, k} x_{0}-x^{*}\right\| \\
& =\left\|\lambda_{k}\left(T_{k} U_{n, k-1} x_{0}-x^{*}\right)+\left(1-\lambda_{k}\right)\left(U_{n, k-1} x_{0}-x^{*}\right)\right\| \\
& =\left\|\lambda_{k}\left(T_{k} x_{0}-x^{*}\right)+\left(1-\lambda_{k}\right)\left(x_{0}-x^{*}\right)\right\| \\
& \leq \lambda_{k}\left\|T_{k} x_{0}-x^{*}\right\|+\left(1-\lambda_{k}\right)\left\|x_{0}-x^{*}\right\| \\
& \leq\left\|x_{0}-x^{*}\right\| .
\end{aligned}
$$

It follows that

$$
\begin{aligned}
\left\|x_{0}-x^{*}\right\| & =\lim _{n \rightarrow \infty}\left\|K_{n} x_{0}-x^{*}\right\| \\
& =\left\|\lambda_{k}\left(T_{k} x_{0}-x^{*}\right)+\left(1-\lambda_{k}\right)\left(x_{0}-x^{*}\right)\right\| \\
& \leq \lambda_{k}\left\|T_{k} x_{0}-x^{*}\right\|+\left(1-\lambda_{k}\right)\left\|x_{0}-x^{*}\right\| \\
& \leq\left\|x_{0}-x^{*}\right\|,
\end{aligned}
$$

which implies

$$
\left\|x_{0}-x^{*}\right\|=\left\|T_{k} x_{0}-x^{*}\right\|=\left\|\lambda_{k}\left(T_{k} x_{0}-x^{*}\right)+\left(1-\lambda_{k}\right)\left(x_{0}-x^{*}\right)\right\| .
$$

By Lemma 2.10, we have $T_{k} x_{0}=x_{0}$, that is $x_{0} \in F\left(T_{k}\right)$. This implies that $x_{0} \in \bigcap_{i=1}^{\infty} F\left(T_{i}\right)$.

\section{Main result}

Theorem 3.1. Let $H$ be a real Hilbert space, and let $M_{i}: H \rightarrow 2^{H}$ be maximal monotone mappings for every $i=1,2, \ldots, N$. Let $B_{i}: H \rightarrow H$ be a $\delta_{i}$-inverse strongly monotone mapping for every $i=1,2, \ldots, N$ and $\left\{T_{i}\right\}_{i=1}^{\infty}$ an infinite family of nonexpansive mappings from $H$ into itself. Let $A$ be a strongly positive linear-bounded self-adjoint operator with the coefficient $0<\bar{\gamma}<1$. Let $G_{i}: H \rightarrow H$ be defined by $J_{M_{i, \eta}}\left(I-\eta B_{i}\right) x=G_{i} x$ for every $x \in H$ and $\eta \in\left(0,2 \delta_{i}\right)$ for every $i=1,2, \ldots, N$ and let $v_{j}=\left(\alpha_{1}^{j}, \alpha_{2}^{j}, \alpha_{3}^{j}\right) \in I \times I \times I, j=1,2,3, \ldots, N$, where $I=\left[\begin{array}{ll}0,1 & 1\end{array}, \alpha_{1}^{j}+\alpha_{2}^{j}+\alpha_{3}^{j}=1\right.$, $\alpha_{1}^{j} \in(0,1)$ for all $j=1,2, \ldots, N-1, \alpha_{1}^{N} \in(0,1] \alpha_{2}^{j}, \alpha_{3}^{j} \in[0,1)$ for all $j=1,2, \ldots, N$.. Let $S: C$ $\rightarrow C$ be the S-mapping generated by $G_{1}, G_{2}, \ldots, G_{N}$ and $v_{1}, v_{2}, \ldots, v_{N}$. Let $\lambda_{1}, \lambda_{2}, \ldots$, be real numbers such that $0<\lambda_{i}<1$ for every $i=1,2, \ldots$, with $\sum_{i=1}^{\infty} \lambda_{i}<\infty$, and let $K_{n}$ be the K-mapping generated by $T_{1}, T_{2}, \ldots, T_{n}$ and $\lambda_{1}, \lambda_{2}, \ldots, \lambda_{n}$, and let $K$ be the $K$-mapping generated by $T_{1}, T_{2}, \ldots$, and $\lambda_{1}, \lambda_{2}, \ldots$, i.e.,

$$
K x=\lim _{n \rightarrow \infty} K_{n} x
$$

for every $x \in C$. Assume that $\mathfrak{F}=\bigcap_{i=1}^{\infty} F\left(T_{i}\right) \bigcap \bigcap_{i=1}^{N} V\left(H, B_{i}, M_{i}\right) \neq \emptyset$. For every $n \in$ $\mathbb{N}, i=1,2, \ldots, N$, let $x_{1} \in H$ and $\left\{x_{n}\right\}$ be the sequence generated by

$$
x_{n+1}=\alpha_{n} \gamma f\left(x_{n}\right)+\beta_{n} x_{n}+\left(\left(1-\beta_{n}\right) I-\alpha_{n} A\right)\left(\gamma_{n} K_{n} x_{n}+\left(1-\gamma_{n}\right) S x_{n}\right),
$$

where $f: H \rightarrow H$ is contractive mapping with coefficient $\theta \in(0,1)$ and $0<\gamma<\frac{\bar{\gamma}}{\theta}$. Let $\left\{\alpha_{n}\right\},\left\{\beta_{n}\right\},\left\{\gamma_{n}\right\}$ be sequences in $[0,1]$, satisfying the following conditions: 
(i) $\lim _{n \rightarrow \infty} \alpha_{n}=0_{\text {and }} \Sigma_{n=0}^{\infty} \alpha_{n}=\infty$

(ii) $0<\liminf _{n \rightarrow \infty} \beta_{n} \leq \limsup _{n \rightarrow \infty} \beta_{n}<1$,

(iii) $\lim _{n \rightarrow \infty} \gamma_{n}=c \in(0,1)$

Then $\left\{x_{n}\right\}$ converges strongly to $z \in \mathfrak{F}$, which solves uniquely the following variational inequality:

$$
\left\langle(A-\gamma f) z, z-x^{*}\right\rangle \leq 0, \quad \forall x^{*} \in \mathfrak{F} .
$$

Equivalently, we have $P_{\mathfrak{F}}(I-A+\gamma f) z=z$.

Proof. Let $z$ be the unique solution of (3.2). First, we will show that the mapping $G_{i}$ is a nonexpansive mapping for every $i=1,2, \ldots, N$. Let $x, y \in H$, since $B_{i}$ is $\delta_{i}$ - inverse strongly monotone mapping and $0<\eta<2 \delta_{i}$, for every $i=1,2, \ldots, N$, we have

$$
\begin{aligned}
\left\|\left(I-\eta B_{i}\right) x-\left(I-\eta B_{i}\right) y\right\|^{2} & =\left\|x-y-\eta\left(B_{i} x-B_{i} y\right)\right\|^{2} \\
& =\|x-y\|^{2}-2 \eta\left\langle x-y, B_{i} x-B_{i} y\right\rangle+\eta^{2}\left\|B_{i} x-B_{i} y\right\|^{2} \\
& \leq\|x-y\|^{2}-2 \delta_{i} \eta\left\|B_{i} x-B_{i} y\right\|^{2}+\eta^{2}\left\|B_{i} x-B_{i} y\right\|^{2} \\
& =\|x-y\|^{2}+\eta\left(\eta-2 \delta_{i}\right)\left\|B_{i} x-B_{i} y\right\|^{2} \\
& \leq\|x-y\|^{2} .
\end{aligned}
$$

Thus, $\left(I-\eta B_{i}\right)$ is a nonexpansive mapping for every $i=1,2, \ldots, N$. By Lemma 2.9, we have $G_{i}=J_{M_{i, \eta}}\left(I-\eta B_{i}\right)$ is a nonexpansive mappings for every $i=1,2, \ldots, N$. Let $x^{*} \in \mathfrak{F}$; by Lemma 2.8 , we have

$$
x^{*}=G_{i} x^{*}=J_{M_{i}, \eta}\left(I-\eta B_{i}\right) x^{*}, \forall i=1,2, \ldots N .
$$

Let $e_{n}=\gamma_{n} K_{n} x_{n}+\left(1-\gamma_{n}\right) S x_{n}$. Since $G_{i}$ is a nonexpansive mapping for every $i=1$, $2, \ldots, N$, we have that $S$ is a nonexpansive mapping. By nonexpansiveness of $K_{n}$ we have

$$
\begin{aligned}
\left\|e_{n}-x^{*}\right\| & =\left\|\gamma_{n}\left(K_{n} x_{n}-x^{*}\right)+\left(1-\gamma_{n}\right)\left(S x_{n}-x^{*}\right)\right\| \\
& \leq \gamma_{n}\left\|K_{n} x_{n}-x^{*}\right\|+\left(1-\gamma_{n}\right)\left\|S x_{n}-x^{*}\right\| \\
& \leq \gamma_{n}\left\|x_{n}-x^{*}\right\|+\left(1-\gamma_{n}\right)\left\|x_{n}-x^{*}\right\| \\
& \leq\left\|x_{n}-x^{*}\right\| .
\end{aligned}
$$

Without loss of generality, by conditions $(i)$ and (ii), we have $\alpha_{n} \leq\left(1-\beta_{n}\right)\|A\|^{-1}$. Since $A$ is a strongly positive linear-bounded self-adjoint operator, we have

$$
\|A\|=\sup \{|\langle A x, x\rangle|: x \in H,\|x\|=1\} .
$$

For each $x \in C$ with $\|x\|=1$, we have

$$
\left\langle\left(\left(1-\beta_{n}\right) I-\alpha_{n} A\right) x, x\right\rangle=1-\beta_{n}-\alpha_{n}\langle A x, x\rangle \geq 1-\beta_{n}-\alpha_{n}\|A\| \geq 0,
$$

then (1 - $\left.\beta_{n}\right) I-\alpha_{n} A$ is positive. By (3.6) and (3.7), we have

$$
\begin{aligned}
\left\|\left(1-\beta_{n}\right) I-\alpha_{n} A\right\| & =\sup \left\{\left\langle\left(\left(1-\beta_{n}\right) I-\alpha_{n} A\right) x, x\right\rangle: x \in C,\|x\|=1\right\} \\
& =\sup \left\{\left\langle 1-\beta_{n}-\alpha_{n}\langle A x, x\rangle: x \in C,\|x\|=1\right\}\right. \\
& \leq 1-\beta_{n}-\alpha_{n}\langle A x, x\rangle \\
& \leq 1-\beta_{n}-\alpha_{n} \bar{\gamma} .
\end{aligned}
$$

We shall divide our proof into six steps. 
Step 1. We will show that the sequence $\left\{x_{n}\right\}$ is bounded. Let $x^{*} \in \mathfrak{F}$, by (3.5) and (3.8), we have

$$
\begin{aligned}
\left\|x_{n+1}-x^{*}\right\|= & \left\|\alpha_{n} \gamma f\left(x_{n}\right)+\beta_{n} x_{n}+\left(\left(1-\beta_{n}\right) I-\alpha_{n} A\right) e_{n}-x^{*}\right\| \\
= & \| \alpha_{n} \gamma f\left(x_{n}\right)-\alpha_{n} A x^{*}+\alpha_{n} A x^{*}-\beta_{n} x^{*}+\beta_{n} x^{*}+\beta_{n} x_{n} \\
& +\left(\left(1-\beta_{n}\right) I-\alpha_{n} A\right) e_{n}-x^{*} \| \\
\leq & \alpha_{n}\left\|\gamma f\left(x_{n}\right)-A x^{*}\right\|+\beta_{n}\left\|x_{n}-x^{*}\right\|+\left\|\left(\left(1-\beta_{n}\right) I-\alpha_{n} A\right)\left(e_{n}-x^{*}\right)\right\| \\
\leq & \alpha_{n}\left\|\gamma f\left(x_{n}\right)-A x^{*}\right\|+\beta_{n}\left\|x_{n}-x^{*}\right\|+\left(\left(1-\beta_{n}\right) I-\alpha_{n} \bar{\gamma}\right)\left\|x_{n}-x^{*}\right\| \\
\leq & \alpha_{n}\left(\left\|\gamma f\left(x_{n}\right)-\gamma f\left(x^{*}\right)\right\|+\left\|\gamma f\left(x^{*}\right)-A x^{*}\right\|\right)+\left(1-\alpha_{n} \bar{\gamma}\right)\left\|x_{n}-x^{*}\right\| \\
\leq & \alpha_{n} \gamma \theta\left\|x_{n}-x^{*}\right\|+\alpha_{n}\left\|\gamma f\left(x^{*}\right)-A x^{*}\right\|+\left(1-\alpha_{n} \bar{\gamma}\right)\left\|x_{n}-x^{*}\right\| \\
= & \alpha_{n}\left\|\gamma f\left(x^{*}\right)-A x^{*}\right\|+\left(1-\alpha_{n}(\bar{\gamma}-\gamma \theta)\right)\left\|x_{n}-x^{*}\right\| \\
\leq & \max \left\{\left\|x_{n}-x^{*}\right\|, \frac{\left\|\gamma f\left(x^{*}\right)-A x^{*}\right\|}{\bar{\gamma}-\gamma \theta}\right\} .
\end{aligned}
$$

By induction, we can prove that $\left\{x_{n}\right\}$ is bounded, and so are $\left\{e_{n}\right\},\left\{K_{n} x_{n}\right\},\left\{S x_{n}\right\}$ and $\left\{G_{i}\right.$ $\left.\left(x_{n}\right)\right\}$ for every $i=1,2, \ldots, N$. Without loss of generality, we can assume that there exists a bounded set $D \subset H$ such that

$$
e_{n}, x_{n}, S x_{n}, K_{n} x_{n}, G_{i} x_{n} \in D, \forall n \in \mathbb{N} \text { and } i=1,2, \ldots, N .
$$

Step 2. We will show that $\lim _{n \rightarrow \infty}\left\|x_{n+1}-x_{n}\right\|=0$.

Define sequence $\left\{z_{n}\right\}$ by $z_{n}=\frac{1}{1-\beta_{n}}\left(x_{n+1}-\beta_{n} x_{n}\right)$.

Then $x_{n+1}=\beta_{n} x_{n}+\left(1-\beta_{n}\right) z_{n}$.

Since $\left\{x_{n}\right\}$ is bounded, we have

$$
\begin{aligned}
\left\|z_{n+1}-z_{n}\right\|= & \left\|\frac{x_{n+2}-\beta_{n+1} x_{n+1}}{1-\beta_{n+1}}-\left(\frac{x_{n+1}-\beta_{n} x_{n}}{1-\beta_{n}}\right)\right\| \\
= & \| \frac{\alpha_{n+1} \gamma f\left(x_{n+1}\right)+\left(\left(1-\beta_{n+1}\right) I-\alpha_{n+1} A\right) e_{n+1}}{1-\beta_{n+1}} \\
& -\left(\frac{\alpha_{n} \gamma f\left(x_{n}\right)+\left(\left(1-\beta_{n}\right) I-\alpha_{n} A\right) e_{n}}{1-\beta_{n}}\right) \| \\
\leq & \alpha_{n+1}\left\|\frac{\gamma f\left(x_{n+1}\right)-A e_{n+1}}{1-\beta_{n+1}}\right\|+\left\|e_{n+1}-e_{n}\right\| \\
& +\alpha_{n}\left\|\frac{\gamma f\left(x_{n}\right)-A e_{n}}{1-\beta_{n}}\right\| .
\end{aligned}
$$

By definition of $e_{n}$ and nonexpansiveness of $S$, we have

$$
\begin{aligned}
\left\|e_{n+1}-e_{n}\right\|= & \left\|\gamma_{n+1} K_{n+1} x_{n+1}+\left(1-\gamma_{n+1}\right) S x_{n+1}-\gamma_{n} K_{n} x_{n}-\left(1-\gamma_{n}\right) S x_{n}\right\| \\
= & \| \gamma_{n+1} K_{n+1} x_{n+1}+\left(1-\gamma_{n+1}\right) S x_{n+1}-\gamma_{n+1} K_{n} x_{n}+\gamma_{n+1} K_{n} x_{n} \\
& -\left(1-\gamma_{n+1}\right) S x_{n}+\left(1-\gamma_{n+1}\right) S x_{n}-\gamma_{n} K_{n} x_{n}-\left(1-\gamma_{n}\right) S x_{n} \| \\
= & \| \gamma_{n+1}\left(K_{n+1} x_{n+1}-K_{n} x_{n}\right)+\left(1-\gamma_{n+1}\right)\left(S x_{n+1}-S x_{n}\right) \\
& +\left(\gamma_{n+1}-\gamma_{n}\right) K_{n} x_{n}+\left(\gamma_{n}-\gamma_{n+1}\right) S x_{n} \| \\
\leq & \gamma_{n+1}\left\|K_{n+1} x_{n+1}-K_{n} x_{n}\right\|+\left(1-\gamma_{n+1}\right)\left\|x_{n+1}-x_{n}\right\| \\
& +\left|\gamma_{n+1}-\gamma_{n}\right|\left\|K_{n} x_{n}\right\|+\left|\gamma_{n}-\gamma_{n+1}\right|\left\|S x_{n}\right\| \\
\leq & \gamma_{n+1}\left\|K_{n+1} x_{n+1}-K_{n} x_{n}\right\|+\left(1-\gamma_{n+1}\right)\left\|x_{n+1}-x_{n}\right\| \\
& +2\left|\gamma_{n+1}-\gamma_{n}\right| M,
\end{aligned}
$$


where $M=\max _{n \in \mathbb{N}}\left\{\| K_{n} x_{n}||,|| S x_{n}||\right\}$. Substituting (3.11) into (3.10), we have

$$
\begin{aligned}
\left\|z_{n+1}-z_{n}\right\| \leq & \alpha_{n+1}\left\|\frac{\gamma f\left(x_{n+1}\right)-A e_{n+1}}{1-\beta_{n+1}}\right\|+\alpha_{n}\left\|\frac{\gamma f\left(x_{n}\right)-A e_{n}}{1-\beta_{n}}\right\|+\left\|e_{n+1}-e_{n}\right\| \\
\leq & \alpha_{n+1}\left\|\frac{\gamma f\left(x_{n+1}\right)-A e_{n+1}}{1-\beta_{n+1}}\right\|+\alpha_{n}\left\|\frac{\gamma f\left(x_{n}\right)-A e_{n}}{1-\beta_{n}}\right\| \\
& +\gamma_{n+1}\left\|K_{n+1} x_{n+1}-K_{n} x_{n}\right\|+\left(1-\gamma_{n+1}\right)\left\|x_{n+1}-x_{n}\right\| \\
& +2\left|\gamma_{n+1}-\gamma_{n}\right| M \\
\leq & \alpha_{n+1}\left\|\frac{\gamma f\left(x_{n+1}\right)-A e_{n+1}}{1-\beta_{n+1}}\right\|+\alpha_{n}\left\|\frac{\gamma f\left(x_{n}\right)-A e_{n}}{1-\beta_{n}}\right\| \\
& +\gamma_{n+1}\left(\left\|K_{n+1} x_{n+1}-K_{n+1} x_{n}\right\|+\left\|K_{n+1} x_{n}-K_{n} x_{n}\right\|\right) \\
& +\left(1-\gamma_{n+1}\right)\left\|x_{n+1}-x_{n}\right\|+2\left|\gamma_{n+1}-\gamma_{n}\right| M \\
\leq & \alpha_{n+1}\left\|\frac{\gamma f\left(x_{n+1}\right)-A e_{n+1}}{1-\beta_{n+1}}\right\|+\alpha_{n}\left\|\frac{\gamma f\left(x_{n}\right)-A e_{n}}{1-\beta_{n}}\right\|+\left\|x_{n+1}-x_{n}\right\| \\
& +\left\|K_{n+1} x_{n}-K_{n} x_{n}\right\|+2\left|\gamma_{n+1}-\gamma_{n}\right| M .
\end{aligned}
$$

It implies that

$$
\begin{aligned}
\left\|z_{n+1}-z_{n}\right\|-\left\|x_{n+1}-x_{n}\right\| \leq & \alpha_{n+1}\left\|\frac{\gamma f\left(x_{n+1}\right)-A e_{n+1}}{1-\beta_{n+1}}\right\|+\alpha_{n}\left\|\frac{\gamma f\left(x_{n}\right)-A e_{n}}{1-\beta_{n}}\right\| \\
& +\left\|K_{n+1} x_{n}-K_{n} x_{n}\right\|+2\left|\gamma_{n+1}-\gamma_{n}\right| M .
\end{aligned}
$$

By (2.3), it implies that

$$
K_{n+1} x_{n}-K_{n} x_{n}=\lambda_{n+1}\left(T_{n+1} K_{n} x_{n}-K_{n} x_{n}\right),
$$

since $\lambda_{n} \rightarrow 0$ as $n \rightarrow \infty$, we have

$$
\lim _{n \rightarrow \infty}\left\|K_{n+1} x_{n}-K_{n} x_{n}\right\|=0 .
$$

By (3.13), (3.14) and conditions (i), (iii), we have

$$
\limsup _{n \rightarrow \infty}\left(\left\|z_{n+1}-z_{n}\right\|-\left\|x_{n+1}-x_{n}\right\|\right) \leq 0 .
$$

By Lemma 2.5, we have

$$
\lim _{n \rightarrow \infty}\left\|z_{n}-x_{n}\right\|=0 .
$$

By condition (ii) and (3.16)

$$
\lim _{n \rightarrow \infty}\left\|x_{n+1}-x_{n}\right\|=\lim _{n \rightarrow \infty}\left(1-\beta_{n}\right)\left\|z_{n}-x_{n}\right\|=0 .
$$

Step 3. We will show that

$$
\lim _{n \rightarrow \infty}\left\|e_{n}-x_{n}\right\|=0 \text {. }
$$

Since $x_{n+1}=\alpha_{n} \gamma f+\beta_{n} x_{n}+\left(\left(1-\beta_{n}\right) I-\alpha_{n} A\right) e_{n}$, we have

$$
\begin{aligned}
\left\|x_{n+1}-e_{n}\right\| & =\left\|\alpha_{n}\left(\gamma f\left(x_{n}\right)-A e_{n}\right)+\beta_{n}\left(x_{n}-e_{n}\right)\right\| \\
& \leq \alpha_{n}\left\|\gamma f\left(x_{n}\right)-A e_{n}\right\|+\beta_{n}\left(\left\|x_{n}-x_{n+1}\right\|+\left\|x_{n+1}-e_{n}\right\|\right),
\end{aligned}
$$

it implies that

$$
\left(1-\beta_{n}\right)\left\|x_{n+1}-e_{n}\right\| \leq \alpha_{n}\left\|\gamma f\left(x_{n}\right)-A e_{n}\right\|+\beta_{n}\left\|x_{n}-x_{n+1}\right\|,
$$


and it follows that

$$
\left\|x_{n+1}-e_{n}\right\| \leq \frac{\alpha_{n}}{\left(1-\beta_{n}\right)}\left\|\gamma f\left(x_{n}\right)-A e_{n}\right\|+\frac{\beta_{n}}{\left(1-\beta_{n}\right)}\left\|x_{n}-x_{n+1}\right\| .
$$

By conditions (i), (ii) and (3.17), we have

$$
\lim _{n \rightarrow \infty}\left\|x_{n+1}-e_{n}\right\|=0 .
$$

Since $\left\|e_{n}-x_{n}\right\| \leq\left\|e_{n}-x_{n+1}\right\|+\left\|x_{n+1}-x_{n}\right\|$, by (3.17) and (3.19), we have

$$
\lim _{n \rightarrow \infty}\left\|e_{n}-x_{n}\right\|=0 \text {. }
$$

Step 4. Define a mapping $Q: H \rightarrow H$ by

$$
Q x=c K x+(1-c) S x, \quad \forall x \in H .
$$

We will show that

$$
\lim _{n \rightarrow \infty}\left\|Q x_{n}-x_{n}\right\|=0
$$

Since

$$
\begin{aligned}
\left\|Q x_{n}-e_{n}\right\| & =\left\|c K x_{n}+(1-c) S x_{n}-\gamma_{n} K_{n} x_{n}-\left(1-\gamma_{n}\right) S x_{n}\right\| \\
& \leq\left\|c K x_{n}-\gamma_{n} K_{n} x_{n}\right\|+\left|\gamma_{n}-c\right|\left\|S x_{n}\right\| \\
& =\left\|c K x_{n}-\gamma_{n} K x_{n}+\gamma_{n} K x_{n}-\gamma_{n} K_{n} x_{n}\right\|+\left|\gamma_{n}-c\right|\left\|S x_{n}\right\| \\
& \leq\left|c-\gamma_{n}\right|\left\|K x_{n}\right\|+\gamma_{n}\left\|K x_{n}-K_{n} x_{n}\right\|+\left|\gamma_{n}-c\right|\left\|S x_{n}\right\| \\
& \leq\left|c-\gamma_{n}\right|\left\|K x_{n}\right\|+\sup _{x \in D}\left\{\left\|K x-K_{n} x\right\|\right\}+\left|\gamma_{n}-c\right|\left\|S x_{n}\right\| .
\end{aligned}
$$

By remark 2.12 and condition (iii), we have

$$
\lim _{n \rightarrow \infty}\left\|Q x_{n}-e_{n}\right\|=0 \text {. }
$$

Since $\left\|Q x_{n}-x_{n}\right\| \leq\left\|Q x_{n}-e_{n}\right\|+\left\|e_{n}-x_{n}\right\|$, from (3.22) and (3.18), we have

$$
\lim _{n \rightarrow \infty}\left\|Q x_{n}-x_{n}\right\|=0 .
$$

Step 5. We will show that

$$
\limsup _{n \rightarrow \infty}\left\langle(\gamma f-A) z, x_{n}-z\right\rangle \leq 0,
$$

where $z=P \mathfrak{F}(I-(A-\gamma f)) z$. Let $\left\{x_{n_{j}}\right\}$ be subsequence of $\left\{x_{n}\right\}$ such that

$$
\limsup _{n \rightarrow \infty}\left\langle(\gamma f-A) z, x_{n}-z\right\rangle=\lim _{j \rightarrow \infty}\left\langle(\gamma f-A) z, x_{n_{j}}-z\right\rangle .
$$

Without loss of generality, we may assume that $\left\{x_{n_{j}}\right\}$ converses weakly to some $q \in$ $H$. By nonexpansiveness of $S$ and $K,(3.20)$ and Lemma 2.3, we have that $Q$ is nonexpansive mapping and

$$
F(Q)=F(K) \bigcap F(S) .
$$


Since $J_{M_{i, \eta}}\left(I-\eta B_{i}\right) x=G_{i} x$ for every $x \in H$ and $i=1,2, \ldots N$, by Lemma 2.8 , we have

$$
V I\left(H, B_{i}, M_{i}\right)=F\left(J_{M_{i, \eta}}\left(I-\eta B_{i}\right)\right)=F\left(G_{i}\right), \quad \forall i=1,2, \ldots N .
$$

By Lemma 2.6 and Lemma 2.9, we have

$$
F(S)=\bigcap_{i=1}^{N} F\left(G_{i}\right)=\bigcap_{i=1}^{N} V I\left(H, B_{i}, M_{i}\right)
$$

By Lemma 2.13, we have

$$
F(K)=\bigcap_{i=1}^{\infty} F\left(T_{i}\right)
$$

By (3.25), (3.27), and (3.28), we have

$$
F(Q)=F(K) \bigcap F(S)=\bigcap_{i=1}^{\infty} F\left(T_{i}\right) \bigcap \bigcap_{i=1}^{N} V I\left(H, B_{i}, M_{i}\right)
$$

Since $x_{n_{j}} \rightarrow q$ as $j \rightarrow \infty$, nonexpansiveness of $Q$, (3.21) and Lemma 2.4, we have

$$
q \in F(Q)=\bigcap_{i=1}^{\infty} F\left(T_{i}\right) \bigcap \bigcap_{i=1}^{N} V I\left(H, B_{i}, M_{i}\right)=\mathfrak{F} .
$$

By (3.24) and (3.30), we have

$$
\limsup _{n \rightarrow \infty}\left\langle(\gamma f-A) z, x_{n}-z\right\rangle=\lim _{n \rightarrow \infty}\left\langle(\gamma f-A) z, x_{n_{j}}-z\right\rangle=\langle(\gamma f-A) z, q-z\rangle \leq 0
$$

Step 6. Finally, we will show that $x_{n} \rightarrow z$ as $n \rightarrow \infty$, where $z=P \mathfrak{F}(I-(A-\gamma f)) z$. Since

$$
\begin{aligned}
\left\|x_{n+1}-z\right\|^{2}= & \left\|\alpha_{n} \gamma f\left(x_{n}\right)+\beta_{n} x_{n}+\left(\left(1-\beta_{n}\right) I-\alpha_{n} A\right) e_{n}-z\right\|^{2} \\
= & \left\|\alpha_{n}\left(\gamma f\left(x_{n}\right)-A z\right)+\beta_{n}\left(x_{n}-z\right)+\left(\left(1-\beta_{n}\right) I-\alpha_{n} A\right)\left(e_{n}-z\right)\right\|^{2} \\
\leq & \left\|\beta_{n}\left(x_{n}-z\right)+\left(\left(1-\beta_{n}\right) I-\alpha_{n} A\right)\left(e_{n}-z\right)\right\|^{2}+2 \alpha_{n}\left\langle\gamma f\left(x_{n}\right)-A z, x_{n+1}-z\right\rangle \\
= & \left(\left\|\beta_{n}\left(x_{n}-z\right)+\left(\left(1-\beta_{n}\right) I-\alpha_{n} A\right)\left(e_{n}-z\right)\right\|\right)^{2}+2 \alpha_{n}\left\langle\gamma f\left(x_{n}\right)-A z, x_{n+1}-z\right\rangle \\
\leq & \left(\left\|\beta_{n}\left(x_{n}-z\right)\right\|+\left\|\left(1-\beta_{n}\right) I-\alpha_{n} A\right\|\left\|e_{n}-z\right\|\right)^{2} \\
& +2 \alpha_{n}\left\langle\gamma f\left(x_{n}\right)-\gamma f(z), x_{n+1}-z\right\rangle+2 \alpha_{n}\left\langle\gamma f(z)-A z, x_{n+1}-z\right\rangle \\
\leq & \left(\left\|\beta_{n}\left(x_{n}-z\right)\right\|+\left(1-\beta_{n}-\alpha_{n} \bar{\gamma}\right)\left\|e_{n}-z\right\|\right)^{2} \\
& +2 \alpha_{n} \gamma \theta\left\|x_{n}-z\right\|\left\|x_{n+1}-z\right\|+2 \alpha_{n}\left\langle\gamma f(z)-A z, x_{n+1}-z\right\rangle \\
\leq & \left(\left\|\beta_{n}\left(x_{n}-z\right)\right\|+\left(1-\beta_{n}-\alpha_{n} \bar{\gamma}\right)\left\|x_{n}-z\right\|\right)^{2} \\
& +2 \alpha_{n} \gamma \theta\left\|x_{n}-z\right\|\left\|x_{n+1}-z\right\|+2 \alpha_{n}\left\langle\gamma f(z)-A z, x_{n+1}-z\right\rangle \\
\leq & \left(\left(1-\alpha_{n} \bar{\gamma}\right)\left\|x_{n}-z\right\|\right)^{2}+\alpha_{n} \gamma \theta\left(\left\|x_{n}-z\right\|^{2}+\left\|x_{n+1}-z\right\|^{2}\right) \\
& +2 \alpha_{n}\left\langle\gamma f(z)-A z, x_{n+1}-z\right\rangle \\
\leq & \left(1-2 \alpha_{n} \bar{\gamma}+\alpha_{n} \gamma \theta\right)\left\|x_{n}-z\right\|^{2}+\alpha_{n}^{2} \bar{\gamma}^{2}\left\|x_{n}-z\right\|^{2}+\alpha_{n} \gamma \theta\left\|x_{n+1}-z\right\|^{2} \\
& +2 \alpha_{n}\left\langle\gamma f(z)-A z, x_{n+1}-z\right\rangle,
\end{aligned}
$$


it implies that

$$
\begin{aligned}
\left\|x_{n+1}-z\right\|^{2} \leq & \frac{\left(1-2 \alpha_{n} \bar{\gamma}+\alpha_{n} \gamma \theta\right)}{1-\alpha_{n} \gamma \theta}\left\|x_{n}-z\right\|^{2}+\frac{\alpha_{n}^{2} \bar{\gamma}^{2}}{1-\alpha_{n} \gamma \theta}\left\|x_{n}-z\right\|^{2} \\
& +\frac{2 \alpha_{n}}{1-\alpha_{n} \gamma \theta}\left\langle\gamma f(z)-A z, x_{n+1}-z\right\rangle \\
= & \frac{\left(1-\alpha_{n} \gamma \theta+\alpha_{n} \gamma \theta-2 \alpha_{n} \bar{\gamma}+\alpha_{n} \gamma \theta\right)}{1-\alpha_{n} \gamma \theta}\left\|x_{n}-z\right\|^{2}+\frac{\alpha_{n}^{2} \bar{\gamma}^{2}}{1-\alpha_{n} \gamma \theta}\left\|x_{n}-z\right\|^{2} \\
& +\frac{2 \alpha_{n}}{1-\alpha_{n} \gamma \theta}\left\langle\gamma f(z)-A z, x_{n+1}-z\right\rangle \\
= & \frac{\left(1-\alpha_{n} \gamma \theta-2 \alpha_{n}(\bar{\gamma}-\gamma \theta)\right)}{1-\alpha_{n} \gamma \theta}\left\|x_{n}-z\right\|^{2}+\frac{\alpha_{n}^{2} \bar{\gamma}^{2}}{1-\alpha_{n} \gamma \theta}\left\|x_{n}-z\right\|^{2} \\
& +\frac{2 \alpha_{n}}{1-\alpha_{n} \gamma \theta}\left\langle\gamma f(z)-A z, x_{n+1}-z\right\rangle \\
= & \left(1-\frac{\left.2 \alpha_{n}(\bar{\gamma}-\gamma \theta)\right)}{1-\alpha_{n} \gamma \theta}\left\|x_{n}-z\right\|^{2}+\frac{\alpha_{n}^{2} \bar{\gamma}^{2}}{1-\alpha_{n} \gamma \theta}\left\|x_{n}-z\right\|^{2}\right. \\
& +\frac{2 \alpha_{n}}{1-\alpha_{n} \gamma \theta}\left\langle\gamma f(z)-A z, x_{n+1}-z\right\rangle \\
= & \left(1-\frac{\left.2 \alpha_{n}(\bar{\gamma}-\gamma \theta)\right)}{1-\alpha_{n} \gamma \theta}\left\|x_{n}-z\right\|^{2}+\frac{\alpha_{n}}{1-\alpha_{n} \gamma \theta}\left(\alpha_{n} \bar{\gamma}^{2}\left\|x_{n}-z\right\|^{2}\right.\right. \\
& \left.+2\left\langle\gamma f(z)-A z, x_{n+1}-z\right\rangle\right) \\
= & \left(1-\frac{\left.2 \alpha_{n}(\bar{\gamma}-\gamma \theta)\right)}{1-\alpha_{n} \gamma \theta}\left\|x_{n}-z\right\|^{2}+\frac{2(\bar{\gamma}-\gamma \theta)}{2(\bar{\gamma}-\gamma \theta)} \frac{\alpha_{n}}{1-\alpha_{n} \gamma \theta}\left(\alpha_{n} \bar{\gamma}^{2}\left\|x_{n}-z\right\|^{2}\right.\right. \\
& \left.+2\left\langle\gamma f(z)-A z, x_{n+1}-z\right\rangle\right) \\
= & \left(1-\frac{\left.2 \alpha_{n}(\bar{\gamma}-\gamma \theta)\right)}{1-\alpha_{n} \gamma \theta}\left\|x_{n}-z\right\|^{2}+\frac{2 \alpha_{n}(\bar{\gamma}-\gamma \theta)}{1-\alpha_{n} \gamma \theta}\left(\frac{\alpha_{n} \bar{\gamma}^{2}}{2(\bar{\gamma}-\gamma \theta)}\left\|x_{n}-z\right\|^{2}\right.\right. \\
& \left.+\frac{2}{2(\bar{\gamma}-\gamma \theta)}\left\langle\gamma f(z)-A z, x_{n+1}-z\right\rangle\right), \\
& +\gamma(x)
\end{aligned}
$$

from condition $i$, step 5 and Lemma 2.2, we can conclude that $x_{n} \rightarrow z$ as $n \rightarrow \infty$, where $z=P_{\mathrm{F}}(I-(A-\gamma f)) z$. This completes the proof.

By means of our main result, we have the following results in the framework of Hilbert space. To prove these results, we need definition and lemma as follows:

Definition 3.1. A mapping $T: C \rightarrow C$ is said to be a $\kappa$-strict pseudo-contraction mapping, if there exists $\kappa \in[0,1)$ such that

$$
\|T x-T y\|^{2} \leq\|x-y\|^{2}+\kappa\|(I-T) x-(I-T) y\|^{2}, \quad \forall x, y \in C .
$$

Lemma 3.2. (see [23]) Let $C$ be a nonempty closed convex subset of a real Hilbert space $H$ and $T: C \rightarrow C$ a $\kappa$-strict pseudo-contraction. Define $S: C \rightarrow C$ by $S x=\alpha x+(1-\alpha) T x$, for each $x \in C$. Then, as $\alpha \in[\kappa, 1), S$ is nonexpansive such that $F(S)=F(T)$.

Corollary 3.3. Let $H$ be a real Hilbert space and let $M_{i}: H \rightarrow 2^{H}$ be maximal monotone mappings for every $i=1,2, \ldots N$. Let $B_{i}: H \rightarrow H$ be a $\delta_{i}$-inverse strongly monotone mapping for every $i=1,2, \ldots N$ and $\left\{T_{i}\right\}_{i=1}^{\infty}$ an infinite family of $\kappa_{i}$-strictly pseudo-contractive mappings from $H$ into itself. Define a mapping $T_{\kappa_{i}} b y T_{\kappa_{i}}=\kappa_{i} x+\left(1-\kappa_{i}\right) T_{i} x, \forall x \in H, i \in \mathbb{N}$. Let $A$ be a strongly positive linear-bounded self-adjoint operator with the coefficient $0<\bar{\gamma}<1$. Let $G_{i}: H \rightarrow H$ be defined by $J_{M_{i, \eta}}\left(I-\eta B_{i}\right) x=G_{i} x$ for every $x \in H$ and $\eta \in(0$, $\left.2 \delta_{i}\right)$ for every $i=1,2, \ldots, N$ and let $v_{j}=\left(\alpha_{1}^{j}, \alpha_{2}^{j}, \alpha_{3}^{j}\right) \in I \times I \times I, j=1,2,3, \ldots, N$, where $I=[0$, 1], $\alpha_{1}^{j}+\alpha_{2}^{j}+\alpha_{3}^{j}=1, \alpha_{1}^{j} \in(0,1)$ for all $j=1,2, \ldots, N-1, \alpha_{1}^{N} \in(0,1] \alpha_{2}^{j}, \alpha_{3}^{j} \in[0,1)$ for all $\mathbf{j}=$ $1,2, \ldots, \mathrm{N}$.. Let $S: C \rightarrow C$ be the $S$ - mapping generated by $G_{1}, G_{2}, \ldots, G_{N}$ and $v_{1}, v_{2}, \ldots, v_{N}$. Let 
$\lambda_{1}, \lambda_{2}, \ldots$, be real numbers such that $0<\lambda_{i}<1$ for every $i=1,2, \ldots$, with $\sum_{i=1}^{\infty} \lambda_{i}<\infty$, and let $K_{n}$ be the K-mapping generated by $T_{\kappa_{1}}, T_{\kappa_{2}}, \ldots, T_{\kappa_{n}}$, and $\lambda_{1}, \lambda_{2}, \ldots, \lambda_{n}$, and let $K$ be the $K$-mapping generated by $T_{\kappa_{1}}, T_{\kappa_{2}}, \ldots$, and $\lambda_{1}, \lambda_{2}, \ldots$, i.e.,

$$
K x=\lim _{n \rightarrow \infty} K_{n} x
$$

for every $x \in C$. Assume that $\mathfrak{F}=\bigcap_{i=1}^{\infty} F\left(T_{i}\right) \bigcap \bigcap_{i=1}^{N} V\left(H, B_{i}, M_{i}\right) \neq \emptyset$. For every $n \in$ $\mathbb{N}, i=1,2, \ldots, N$, let $x_{1} \in H$ and $\left\{x_{n}\right\}$ be the sequence generated by

$$
x_{n+1}=\alpha_{n} \gamma f\left(x_{n}\right)+\beta_{n} x_{n}+\left(\left(1-\beta_{n}\right) I-\alpha_{n} A\right)\left(\gamma_{n} K_{n} x_{n}+\left(1-\gamma_{n}\right) S x_{n}\right),
$$

where $f: H \rightarrow H$ is contractive mapping with coefficient $\theta \in(0,1)$ and $0<\gamma<\frac{\bar{\gamma}}{\theta}$. Let $\left\{\alpha_{n}\right\},\left\{\beta_{n}\right\},\left\{\gamma_{n}\right\}$ be sequences in $[0,1]$, satisfying the following conditions:

(i) $\lim _{n \rightarrow \infty} \alpha_{n}=0_{\text {and }} \Sigma_{n=0}^{\infty} \alpha_{n}=\infty$,

(ii) $0<\liminf _{n \rightarrow \infty} \beta_{n} \leq \limsup _{n \rightarrow \infty} \beta_{n}<1$,

(iii) $\lim _{n \rightarrow \infty} \gamma_{n}=c \in(0,1)$.

Then $\left\{x_{n}\right\}$ converges strongly to $z \in \mathfrak{F}$, which solves uniquely the following variational inequality:

$$
\left\langle(A-\gamma f) z, z-x^{*}\right\rangle \leq 0, \quad \forall x^{*} \in \mathfrak{F} .
$$

Equivalently, we have $P_{\mathfrak{F}}(I-A+\gamma f) z=z$.

Proof. For every $i \in \mathbb{N}$, by Lemma 3.2, we have that $T_{\kappa_{i}}$ is a nonexpansive mapping and $\bigcap_{i=1}^{\infty} F\left(T_{\kappa_{i}}\right)=\bigcap_{i=1}^{\infty} F\left(T_{i}\right)$. From Theorem 3.1 and Lemma 2.13, we can reach the desired conclusion.

Corollary 3.4. Let $H$ be a real Hilbert space and let $M: H \rightarrow 2^{H}$ be maximal monotone mappings. Let $B: H \rightarrow H$ be a $\delta$ - inverse strongly monotone mapping and $\left\{T_{i}\right\}_{i=1}^{\infty}$ an infinite family of $\kappa_{i}$-strictly pseudo-contractive map-pings from $H$ into itself. Define a mapping $T_{\kappa_{i}}=\kappa_{i} x+\left(1-\kappa_{i}\right) T_{i} x b y T_{\kappa_{i}}=\kappa_{i} x+\left(1-\kappa_{i}\right) T_{i} x, \forall x \in H, i \in \mathbb{N}$. Let $A$ be a strongly positive linear-bounded self-adjoint operator with the coefficient $0<\bar{\gamma}<1$. Let $\lambda_{1}, \lambda_{2}, \ldots$, be real numbers such that $0<\lambda_{i}<1$ for every $i=1,2, \ldots$, with $\sum_{i=1}^{\infty} \lambda_{i}<\infty$, and let $K_{n}$ be the $K$-mapping generated by $T_{\kappa_{1}}, T_{\kappa_{2}}, \ldots, T_{\kappa_{n}}$ and $\lambda_{1}, \lambda_{2}, \ldots, \lambda_{n}$, and let $K$ be the K-mapping generated by $T_{\kappa_{1}}, T_{\kappa_{2}}, \ldots$, and $\lambda_{1}, \lambda_{2}, \ldots$, i.e.,

$$
K x=\lim _{n \rightarrow \infty} K_{n} x
$$

for every $x \in C$. Assume that $\mathfrak{F}=\bigcap_{i=1}^{\infty} F\left(T_{i}\right) \bigcap V(H, B, M) \neq \emptyset$. For every $n \in \mathbb{N}$, let $x_{1}$ $\in H$ and $\left\{x_{n}\right\}$ be the sequence generated by

$$
x_{n+1}=\alpha_{n} \gamma f\left(x_{n}\right)+\beta_{n} x_{n}+\left(\left(1-\beta_{n}\right) I-\alpha_{n} A\right)\left(\gamma_{n} K_{n} x_{n}+\left(1-\gamma_{n}\right) J_{M, \eta}(I-\eta B) x_{n}(\beta .3 .33)\right.
$$

where $f: H \rightarrow H$ is contractive mapping with coefficient $\theta \in(0,1)$ and $0<\gamma<\frac{\bar{\gamma}}{\theta}$, $\eta$ $\in(0,2 \delta),\left\{\alpha_{n}\right\},\left\{\beta_{n}\right\},\left\{\gamma_{n}\right\}$ are sequences in $[0,1]$, satisfying the following conditions:

(i) $\lim _{n \rightarrow \infty} \alpha_{n}=0_{\text {and }} \Sigma_{n=0}^{\infty} \alpha_{n}=\infty$, 
(ii) $0<\liminf _{n \rightarrow \infty} \beta_{n} \leq \limsup _{n \rightarrow \infty} \beta_{n}<1$,

(iii) $\lim _{n \rightarrow \infty} \gamma_{n}=c \in(0,1)$.

Then $\left\{x_{n}\right\}$ converges strongly to $z \in \mathfrak{F}$, which solves uniquely the following variational inequality

$$
\left\langle(A-\gamma f) z, z-x^{*}\right\rangle \leq 0, \quad \forall x^{*} \in \mathfrak{F} .
$$

Equivalently, we have $P_{\mathfrak{F}}(I-A+\gamma f) z=z$.

Proof. Putting $N=1$ in Corollary 3.3, we can reach the desired conclusion.

\section{Competing interests}

The authors declare that they have no competing interests.

\section{Received: 24 February 2011 Accepted: 18 August 2011 Published: 18 August 2011}

\section{References}

1. Goebel, K, Kirk, WA: Topics in Metric Fixed Point Theory. In Cambridge Stud. Adv. Math, vol. 28,Cambridge University Press, Cambridge (1990)

2. Bauschke, HH, Borwein, JM: On projection algorithms for solving convex feasibility problems. SIAM Rev. 38, 367-426 (1996). doi:10.1137/S0036144593251710

3. Combettes, PL: The foundations of set theoretic estimation. Proc. IEEE. 81, 182-208 (1993)

4. liduka, H, Takahashi, W: Weak convergence theorem by Ces'aro means for nonexpansive mappings and inverse-strongly monotone mappings. J. Nonlinear Convex Anal. 7, 105-113 (2006)

5. Kangtunyakarn, A, Suantai, S: Hybrid iterative scheme for generalized equilibrium problems and fixed point problems of finite family of nonexpansive mappings. Nonlinear Anal.: Hybrid Systems. 3, 296-309 (2009). doi:10.1016/j. nahs.2009.01.012

6. Verma, RU: Generalized system for relaxed cocoercive variational inequalities and its projection methods. J. Optim Theory Appl. 121, 203-210 (2004)

7. Blum, E, Oettli, W: From optimization and variational inequalities to equilibrium problems. Math. Stud. 63, 123-145 (1994)

8. Zeng, LC, Wu, SY, Yao, JC: Generalized KKM theorem with applications to generalized minimax inequalities and generalized equilibrium problems. Taiwan. J. Math. 10(6), 1497-1514 (2006)

9. Qin, X, Shang, M, Zhou, H: Strong convergence of a general iterative method for variational inequality problems and fixed point problems in Hilbert spaces. Appl. Math. Commun. 200, 242-253 (2008). doi:10.1016/j.amc.2007.11.004

10. Marino, G, Xu, HK: A general iterative method for nonexpansive mappings in Hilbert spaces. J. Math. Anal. Appl. 318, 43-52 (2006). doi:10.1016/j.jmaa.2005.05.028

11. Zhang, SS, Lee, JHW, Chan, CK: Algorithms of common solutions for quasi variational inclusion and fixed point problems. Appl. Math. Mech. 29, 571-581 (2008). doi:10.1007/s10483-008-0502-y

12. Chang, SS: Set-valued variational inclusions in Banach spaces. J. Math. Anal. Appl. 248, 438-454 (2000). doi:10.1006/ jmaa.2000.6919

13. Noor, MA, Noor, Kl: Sensitivity analysis for quasi-variational inclusions. J. Math. Anal. Appl. 236, $290-299$ (1999). doi:10.1006/jmaa.1999.6424

14. Takahashi, W, Toyoda, M: Weak convergence theorems for nonexpansive mappings and monotone mappings. J. Optim. Theor. Appl. 118, 417-428 (2003). doi:10.1023/A:1025407607560

15. Li, Y, Wu, C: On the Convergence for an iterative method for quasivariational inclusions. Fixed Point Theory Appl 2010 , 11 (2010). Article ID 278973

16. Hao, Y: On variational inclusion and common fixed point problems in Hilbert spaces with applications. Appl. Math. Comput. 217(7), 3000-3010 (2010). doi:10.1016/j.amc.2010.08.033

17. Takahashi, W: Nonlinear Functional Analysis. Yokohama Publishers, Yokohama (2000)

18. Xu, HK: Iterative algorithms for nonlinear opearators. J. Lond. Math. Soc. 66, 240-256 (2002). doi:10.1112/ S0024610702003332

19. Bruck, RE: Properties of fixed point sets of nonexpansive mappings in Banach spaces. Trans. Am. Math. Soc. 179, 251-262 (1973)

20. Browder, FE: Nonlinear operators and nonlinear equations of evolution in Banach spaces. Proc. Sympos. Pure. Math. 18, 78-81 (1976)

21. Suzuki, T: Strong convergence of Krasnoselskii and Manns type sequences for one-parameter nonexpansive semigroups without Bochner integrals. J. Math. Anal. Appl. 305, 227-239 (2005). doi:10.1016/j.jmaa.2004.11.017

22. Chang, SS, Lee, HWJ, Chan, CK: A new method for solving equilibrium problem fixed point problem and variational inequality problem with application to optimization. Nonlinear Anal. 70, 3307-3319 (2009). doi:10.1016/j.na.2008.04.035

23. Zhou, Y: Convergence theorems of fixed points for k-strict pseudocontractions in Hilbert spaces. Nonlinear Anal. 69, 456-462 (2008). doi:10.1016/j.na.2007.05.032

doi:10.1186/1687-1812-2011-38

Cite this article as: Kangtunyakarn: Iterative algorithms for finding a common solution of system of the set of variational inclusion problems and the set of fixed point problems. Fixed Point Theory and Applications 2011 2011:38. 